\title{
Modelling Real World Using Stochastic Processes and Filtration
}

\author{
Peter Jaeger \\ Siegmund-Schacky-Str. 18a \\ 80993 Munich, Germany
}

Summary. First we give an implementation in Mizar 2] basic important definitions of stochastic finance, i.e. filtration (9], pp. 183 and 185), adapted stochastic process (9, p. 185) and predictable stochastic process (6], p. 224). Second we give some concrete formalization and verification to real world examples.

In article [8] we started to define random variables for a similar presentation to the book [6]. Here we continue this study. Next we define the stochastic process. For further definitions based on stochastic process we implement the definition of filtration.

To get a better understanding we give a real world example and connect the statements to the theorems. Other similar examples are given in [10, pp. 143159 and in 12, pp. 110-124. First we introduce sets which give informations referring to today $\left(\Omega_{\text {now }}\right.$, Def.6), tomorrow $\left(\Omega_{\text {fut }}\right.$, Def.7) and the day after tomorrow $\left(\Omega_{f u t_{2}}\right.$, Def.8). We give an overview for some events in the $\sigma$-algebras $\Omega_{n o w}, \Omega_{f u t 1}$ and $\Omega_{f u t 2}$, see theorems (22) and (23).

The given events are necessary for creating our next functions. The implementations take the form of: $\Omega_{\text {now }} \subset \Omega_{\text {fut } 1} \subset \Omega_{\text {fut } 2}$ see theorem (24). This tells us growing informations from now to the future $1=$ now, $2=$ tomorrow, $3=$ the day after tomorrow.

We install functions $f:\{1,2,3,4\} \rightarrow \mathbb{R}$ as following:

$f_{1}: x \rightarrow 100, \forall x \in \operatorname{dom} f$, see theorem (36),

$f_{2}: x \rightarrow 80$, for $x=1$ or $x=2$ and

$f_{2}: x \rightarrow 120$, for $x=3$ or $x=4$, see theorem (37),

$f_{3}: x \rightarrow 60$, for $x=1, f_{3}: x \rightarrow 80$, for $x=2$ and

$f_{3}: x \rightarrow 100$, for $x=3, f_{3}: x \rightarrow 120$, for $x=4$ see theorem (38).

These functions are real random variable: $f_{1}$ over $\Omega_{\text {now }}, f_{2}$ over $\Omega_{f u t 1}, f_{3}$ over $\Omega_{f u t 2}$, see theorems (46), (43) and (40). We can prove that these functions can be used for giving an example for an adapted stochastic process. See theorem (49). 
We want to give an interpretation to these functions: suppose you have an equity $A$ which has now $\left(=w_{1}\right)$ the value 100 . Tomorrow $A$ changes depending which scenario occurs - e.g. another marketing strategy. In scenario $1\left(=w_{11}\right)$ it has the value 80 , in scenario $2\left(=w_{12}\right)$ it has the value 120 . The day after tomorrow $A$ changes again. In scenario $1\left(=w_{111}\right)$ it has the value 60 , in scenario $2\left(=w_{112}\right)$ the value 80 , in scenario $3\left(=w_{121}\right)$ the value 100 and in scenario 4 $\left(=w_{122}\right)$ it has the value 120 . For a visualization refer to the tree:

$$
\begin{aligned}
& \text { Now tomorrow the day after tomorrow } \\
& w_{111}=\{1\} \\
& w_{11}=\{1,2\}<w_{112}=\{2\} \\
& w_{1}=\{1,2,3,4\} \quad< \\
& w_{121}=\{3\} \\
& w_{12}=\{3,4\}<w_{122}=\{4\}
\end{aligned}
$$

The sets $w_{1}, w_{11}, w_{12}, w_{111}, w_{112}, w_{121}, w_{122}$ which are subsets of $\{1,2,3,4\}$, see (22), tell us which market scenario occurs. The functions tell us the values to the relevant market scenario:

$$
\begin{aligned}
& \text { Now tomorrow the day after tomorrow } \\
& f_{3}\left(w_{i}\right)=60, w_{i} \text { in } w_{111} \\
& \begin{array}{l}
f_{2}\left(w_{i}\right)=80< \\
w_{i} \text { in } w_{11}
\end{array} f_{3}\left(w_{i}\right)=80, w_{i} \text { in } w_{112} \\
& f_{1}\left(w_{i}\right)=100< \\
& w_{i} \text { in } w_{1} \\
& f_{3}\left(w_{i}\right)=100, w_{i} \text { in } w_{121} \\
& \begin{array}{l}
f_{2}\left(w_{i}\right)=120< \\
w_{i} \text { in } w_{12}
\end{array} f_{3}\left(w_{i}\right)=120, w_{i} \text { in } w_{122}
\end{aligned}
$$

For a better understanding of the definition of the random variable and the relation to the functions refer to [7, p. 20. For the proof of certain sets as $\sigma$-fields refer to [7, pp. 10-11 and [9], pp. 1-2.

This article is the next step to the arbitrage opportunity. If you use for example a simple probability measure, refer, for example to literature [3], pp. 28-34, 6], p. 6 and p. 232 you can calculate whether an arbitrage exists or not. Note, that the example given in literature [3] needs 8 instead of 4 informations as in our model. If we want to code the first 3 given time points into our model we would have the following graph, see theorems (47), (44) and (41):

$$
\begin{array}{lll}
\text { Now } & \begin{array}{l}
\text { tomorrow } \\
f_{2}\left(w_{i}\right)=150<\begin{array}{l}
\text { the day after tomorrow } \\
f_{3} \text { in } w_{11}
\end{array} \\
f_{1}\left(w_{i}\right)=125<180, w_{i} \text { in } w_{111}
\end{array} \\
w_{i} \text { in } w_{1} & f_{3}\left(w_{i}\right)=120, w_{i} \text { in } w_{112} \\
& f_{2}\left(w_{i}\right)=100<\begin{array}{l}
f_{3}\left(w_{i}\right)=120, w_{i} \text { in } w_{121} \\
w_{i} \text { in } w_{12}
\end{array} \\
& & f_{3}\left(w_{i}\right)=80, w_{i} \text { in } w_{122}
\end{array}
$$

The function for the "Call-Option" is given in literature [3], p. 28. The function is realized in Def.5. As a background, more examples for using the definition of filtration are given in 9], pp. 185-188. 
MSC: 60G05 03B35

Keywords: stochastic process; random variable

MML identifier: FINANCE3, version: 8.1.04 5.36.1267

\section{Preliminaries}

Now we state the proposition:

(1) Let us consider objects $a, b$. If $a \neq b$, then $\{a\} \subset\{a, b\}$.

Let $I$ be a non empty subset of $\mathbb{N}$. Observe that $I\left(\in 2^{\mathbb{R}}\right)$ is non empty.

Let us consider an element $T$ of $\mathbb{N}$. Now we state the propositions:

(2) $\{w$, where $w$ is an element of $\mathbb{N}: w>0$ and $w \leqslant T\} \subseteq\{w$, where $w$ is an element of $\mathbb{N}: w \leqslant T\}$.

(3) $\{w$, where $w$ is an element of $\mathbb{N}: w \leqslant T\}$ is a non empty subset of $\mathbb{N}$.

(4) If $T>0$, then $\{w$, where $w$ is an element of $\mathbb{N}: w>0$ and $w \leqslant T\}$ is a non empty subset of $\mathbb{N}$.

Proof: $\{w$, where $w$ is an element of $\mathbb{N}: w>0$ and $w \leqslant T\}$ is a subset of $\mathbb{N} .1>0$ and $1 \leqslant T$ by [1, (24)].

Now we state the proposition:

(5) Let us consider a non empty set $\Omega$. Then $\Omega \longmapsto 1$ is a function from $\Omega$ into $\mathbb{R}$.

\section{Special Random Variables}

Now we state the proposition:

(6) Let us consider a natural number $d$, a sequence $\varphi$ of real numbers, a non empty set $\Omega$, a $\sigma$-field $F$ of subsets of $\Omega$, a non empty set $X$, a sequence $G$ of $X$, and an element $w$ of $\Omega$. Then the portfolio value for future extension of $d, \varphi, F, G$ and $w\}$ is an event of the Borel sets.

Let $d$ be a natural number, $\varphi$ be a sequence of real numbers, $\Omega$ be a non empty set, $F$ be a $\sigma$-field of subsets of $\Omega, X$ be a non empty set, $G$ be a sequence of $X$, and $w$ be an element of $\Omega$. Note that the portfolio value for future extension of $d, \varphi, F, G$ and $w$ yields an element of $\mathbb{R}$. The $\mathcal{R} \mathcal{V}$-portfolio value for future extension of $\varphi, F, G$ and $d$ yielding a function from $\Omega$ into $\mathbb{R}$ is defined by

(Def. 1) for every element $w$ of $\Omega$,it $(w)=$ the portfolio value for future extension of $d, \varphi, F, G$ and $w$. 
Let us observe that the $\mathcal{R} \mathcal{V}$-portfolio value for future extension of $\varphi, F, G$ and $d$ yields a random variable of $F$ and the Borel sets. Let $w$ be an element of $\Omega$. Let us note that the portfolio value for future of $d, \varphi, F, G$ and $w$ yields a real number and is defined by the term

(Def. 2) $\quad\left(\sum_{\alpha=0}^{\kappa}((\right.$ the elements of the random variables for the future elements of portfolio value of $(\varphi, F, G, w)) \uparrow 1)(\alpha))_{\kappa \in \mathbb{N}}(d-1)$.

Let us note that the portfolio value for future of $d, \varphi, F, G$ and $w$ yields an element of $\mathbb{R}$. The $\mathcal{R} \mathcal{V}$-portfolio value for future of $\varphi, F, G$ and $d$ yielding a function from $\Omega$ into $\mathbb{R}$ is defined by

(Def. 3) for every element $w$ of $\Omega$,it $(w)=$ the portfolio value for future of $d+1$, $\varphi, F, G$ and $w$.

Let us note that the $\mathcal{R} \mathcal{V}$-portfolio value for future of $\varphi, F, G$ and $d$ yields a random variable of $F$ and the Borel sets. Now we state the propositions:

(7) Let us consider a natural number $d$, a sequence $\varphi$ of real numbers, a non empty set $\Omega$, a $\sigma$-field $F$ of subsets of $\Omega$, a non empty set $X$, a sequence $G$ of $X$, and an element $w$ of $\Omega$. Then

(i) the portfolio value for future of $d+1, \varphi, F, G$ and $w=($ the $\mathcal{R} \mathcal{V}$-portfolio value for future of $\varphi, F, G$ and $d)(w)$, and

(ii) $\{$ the portfolio value for future of $d+1, \varphi, F, G$ and $w\}$ is an event of the Borel sets.

(8) Let us consider a non empty set $\Omega$, a $\sigma$-field $F$ of subsets of $\Omega$, a non empty set $X$, a sequence $G$ of $X$, a sequence $\varphi$ of real numbers, and a natural number $d$. Then the $\mathcal{R} \mathcal{V}$-portfolio value for future extension of $\varphi, F, G$ and $d+1=($ the $\mathcal{R} \mathcal{V}$-portfolio value for future of $\varphi, F, G$ and $d)+$ (the random variables for the future elements of portfolio value of $(\varphi, F, G, 0))$.

(9) Let us consider non empty sets $\Omega, \Omega_{2}$, a $\sigma$-field $\Sigma$ of subsets of $\Omega$, a $\sigma$ field $\Sigma_{2}$ of subsets of $\Omega_{2}$, and an element $s$ of $\Omega_{2}$. Then $\Omega \longmapsto s$ is random variable on $\Sigma$ and $\Sigma_{2}$.

(10) Let us consider a non empty set $\Omega$, a $\sigma$-field $\Sigma$ of subsets of $\Omega$, a random variable $\mathcal{R} \mathcal{V}$ of $\Sigma$ and the Borel sets, and an element $K$ of $\mathbb{R}$. Then $\mathcal{R} \mathcal{V}-$ $(\Omega \longmapsto K)$ is a random variable of $\Sigma$ and the Borel sets. The theorem is a consequence of (9).

Let $\Omega$ be a non empty set, $\mathcal{R} \mathcal{V}$ be a function from $\Omega$ into $\mathbb{R}$, and $w$ be an element of $\Omega$. The functor Set-Call-Option $(\mathcal{R} \mathcal{V}, w)$ yielding an element of $\mathbb{R}$ is defined by the term

(Def. 4) $\begin{cases}\mathcal{R} \mathcal{V}(w), & \text { if } \mathcal{R} \mathcal{V}(w) \geqslant 0, \\ 0, & \text { otherwise. }\end{cases}$ 
Let $\Sigma$ be a $\sigma$-field of subsets of $\Omega, \mathcal{R} \mathcal{V}$ be a random variable of $\Sigma$ and the Borel sets, and $K$ be an element of $\mathbb{R}$. The Call-Option on $\mathcal{R} \mathcal{V}$ and $K$ yielding a function from $\Omega$ into $\mathbb{R}$ is defined by

(Def. 5) for every element $w$ of $\Omega$, if $(\mathcal{R} \mathcal{V}-(\Omega \longmapsto K))(w) \geqslant 0$, then it $(w)=$ $(\mathcal{R} \mathcal{V}-(\Omega \longmapsto K))(w)$ and if $(\mathcal{R} \mathcal{V}-(\Omega \longmapsto K))(w)<0$, then it $(w)=0$.

\section{SPECIAL $\sigma$-FIELDS}

Let us consider a sequence $A_{1}$ of subsets of $\{1,2,3,4\}$ and a real number $w$. Now we state the propositions:

(11) Suppose $w=1$ or $w=3$. Then suppose for every natural number $n$, $A_{1}(n)=\emptyset$ or $A_{1}(n)=\{1,2\}$ or $A_{1}(n)=\{3,4\}$ or $A_{1}(n)=\{1,2,3,4\}$. Then $\{w\} \neq$ Intersection $A_{1}$.

(12) Suppose $w=2$ or $w=4$. Then suppose for every natural number $n$, $A_{1}(n)=\emptyset$ or $A_{1}(n)=\{1,2\}$ or $A_{1}(n)=\{3,4\}$ or $A_{1}(n)=\{1,2,3,4\}$. Then $\{w\} \neq$ Intersection $A_{1}$.

Now we state the propositions:

(13) Let us consider sets $M, A_{1}, A_{2}$. Suppose $M=\{\emptyset,\{1,2,3,4\}\}$ and $A_{1}$, $A_{2} \in M$. Then $A_{1} \cap A_{2} \in M$.

(14) Let us consider a sequence $A_{1}$ of subsets of $\{1,2,3,4\}$. Suppose for every natural number $n$ and for every natural number $k, A_{1}(n) \cap A_{1}(k) \neq \emptyset$ and $\operatorname{rng} A_{1} \subseteq\{\emptyset,\{1,2\},\{3,4\},\{1,2,3,4\}\}$. Then

(i) Intersection $A_{1}=\emptyset$, or

(ii) Intersection $A_{1}=\{1,2\}$, or

(iii) Intersection $A_{1}=\{3,4\}$, or

(iv) Intersection $A_{1}=\{1,2,3,4\}$.

Proof: For every natural number $n, A_{1}(n) \in\{\emptyset,\{1,2\},\{3,4\},\{1,2,3,4\}\}$ by [1, (20)], [4, (3)]. For every natural number $n, A_{1}(n)=\emptyset$ or $A_{1}(n)=$ $\{1,2\}$ or $A_{1}(n)=\{3,4\}$ or $A_{1}(n)=\{1,2,3,4\}$.

Let us consider a sequence $A_{1}$ of subsets of $\{1,2,3,4\}$ and a set $M$. Now we state the propositions:

(15) Suppose $M=\{\emptyset,\{1,2\},\{3,4\},\{1,2,3,4\}\}$ and Intersection $A_{1}=\{1,2,3,4\}$. Then Intersection $A_{1} \in M$.

(16) Suppose $M=\{\emptyset,\{1,2\},\{3,4\},\{1,2,3,4\}\}$ and Intersection $A_{1}=\{3,4\}$. Then Intersection $A_{1} \in M$.

(17) Suppose $M=\{\emptyset,\{1,2\},\{3,4\},\{1,2,3,4\}\}$ and Intersection $A_{1}=\{1,2\}$. Then Intersection $A_{1} \in M$. 
(18) Suppose $M=\{\emptyset,\{1,2\},\{3,4\},\{1,2,3,4\}\}$ and Intersection $A_{1}=\emptyset$. Then Intersection $A_{1} \in M$.

Now we state the propositions:

(19) Let us consider a set $M$, and a sequence $A_{1}$ of subsets of $\{1,2,3,4\}$. Suppose $\operatorname{rng} A_{1} \subseteq M$ and $M=\{\emptyset,\{1,2\},\{3,4\},\{1,2,3,4\}\}$.

Then Intersection $A_{1} \in M$.

Proof: Intersection $A_{1} \in\{\emptyset,\{1,2\},\{3,4\},\{1,2,3,4\}\}$ by [11, (13)], (14).

(20) Let us consider sets $M, M_{1}$, and a sequence $A_{1}$ of subsets of $M_{1}$. Suppose $M_{1}=\{1,2,3,4\}$ and $\operatorname{rng} A_{1} \subseteq M$ and $M=\{\emptyset,\{1,2,3,4\}\}$. If Intersection $A_{1} \neq \emptyset$, then Intersection $A_{1} \in M$.

Proof: For every natural number $n, A_{1}(n)=\emptyset$ or $A_{1}(n)=\{1,2,3,4\}$ by [1, (20)], [4, (3)]. If there exists a natural number $n$ such that $A_{1}(n)=\emptyset$, then Intersection $A_{1}=\emptyset$ by [11, (13)]. Intersection $A_{1}=\{1,2,3,4\}$ by [11, (13)].

(21) Let us consider sets $M, M_{1}$, and a sequence $A_{1}$ of subsets of $M_{1}$. Suppose $M_{1}=\{1,2,3,4\}$ and $\operatorname{rng} A_{1} \subseteq M$ and $M=\{\emptyset,\{1,2,3,4\}\}$. Let us consider a natural number $k_{1}$, and a natural number $k_{2}$. Then $A_{1}\left(k_{1}\right) \cap A_{1}\left(k_{2}\right) \in M$. Proof: $k_{1} \in \operatorname{dom} A_{1}$ by [1, (20)]. $k_{2} \in \operatorname{dom} A_{1}$ by [1, (20)]. $A_{1}\left(k_{1}\right) \cap$ $A_{1}\left(k_{2}\right) \in M$.

The functor $\Omega_{\text {now }}$ yielding a $\sigma$-field of subsets of $\{1,2,3,4\}$ is defined by the term

(Def. 6) $\{\emptyset,\{1,2,3,4\}\}$.

The functor $\Omega_{f u t 1}$ yielding a $\sigma$-field of subsets of $\{1,2,3,4\}$ is defined by the term

(Def. 7) $\{\emptyset,\{1,2\},\{3,4\},\{1,2,3,4\}\}$.

The functor $\Omega_{\text {fut } 2}$ yielding a $\sigma$-field of subsets of $\{1,2,3,4\}$ is defined by the term

(Def. 8) $2^{\{1,2,3,4\}}$.

Let us consider a set $\Omega$.

Let us assume that $\Omega=\{1,2,3,4\}$. Now we state the propositions:

(i) $\{1\} \subseteq \Omega$, and

(ii) $\{2\} \subseteq \Omega$, and

(iii) $\{3\} \subseteq \Omega$, and

(iv) $\{4\} \subseteq \Omega$, and

(v) $\{1,2\} \subseteq \Omega$, and

(vi) $\{3,4\} \subseteq \Omega$, and 
(vii) $\emptyset \subseteq \Omega \subseteq \Omega$.

$(23)$

(i) $\Omega, \emptyset \in \Omega_{\text {now }}$, and

(ii) $\{1,2\},\{3,4\}, \Omega, \emptyset \in \Omega_{f u t 1}$, and

(iii) $\Omega, \emptyset,\{1\},\{2\},\{3\},\{4\} \in \Omega_{\text {fut } 2}$.

Now we state the proposition:

(24) $\Omega_{\text {now }} \subset \Omega_{\text {fut } 1} \subset \Omega_{\text {fut } 2}$.

\section{Construction of Filtration and Examples}

Now we state the propositions:

(25) There exists a non empty set $\Omega$ and there exist $\sigma$-fields $F_{1}, F_{2}, F_{3}$ of subsets of $\Omega$ such that $F_{1} \subset F_{2} \subset F_{3}$.

(26) There exist non empty sets $\Omega_{1}, \Omega_{2}, \Omega_{3}, \Omega_{4}$ such that

(i) $\Omega_{1} \subset \Omega_{2} \subset \Omega_{3} \subset \Omega_{4}$, and

(ii) there exists a $\sigma$-field $F_{1}$ of subsets of $\Omega_{1}$ and there exists a $\sigma$-field $F_{2}$ of subsets of $\Omega_{2}$ and there exists a $\sigma$-field $F_{3}$ of subsets of $\Omega_{3}$ and there exists a $\sigma$-field $F_{4}$ of subsets of $\Omega_{4}$ such that $F_{1} \subseteq F_{2} \subseteq F_{3} \subseteq F_{4}$.

Let $I, \Omega$ be non empty sets, $\Sigma$ be a $\sigma$-field of subsets of $\Omega, M$ be a many sorted $\sigma$-field over $I$ and $\Sigma$, and $i$ be an element of $I$. The functor $\mathcal{M}_{\sigma \text {-field }}(M, i)$ yielding a $\sigma$-field of subsets of $\Omega$ is defined by the term

(Def. 9) $M(i)$.

Let $\Omega$ be a non empty set and $I$ be a non empty subset of $\mathbb{R}$.

A filtration of $I$ and $\Sigma$ is a many sorted $\sigma$-field over $I$ and $\Sigma$ and is defined by

(Def. 10) for every elements $s, t$ of $I$ such that $s \leqslant t$ holds $i t(s)$ is a subset of $i t(t)$ and for every element $t$ of $I$, it $(t) \subseteq \Sigma$.

Let $F$ be a filtration of $I$ and $\Sigma$ and $i$ be an element of $I$. The $i-\mathcal{E F}$ of $F$ yielding a $\sigma$-field of subsets of $\Omega$ is defined by the term

(Def. 11) $F(i)$.

Let $k$ be an element of $\{1,2,3\}$. The functor Select $12-\sigma$-field $(k)$ yielding a subset of $2^{\{1,2,3,4\}}$ is defined by the term

(Def. 12) $\begin{cases}\Omega_{\text {now }}, & \text { if } k=1, \\ \Omega_{\text {fut } 1}, & \text { otherwise. }\end{cases}$

The functor Select123- $\sigma$-field $(k)$ yielding a subset of $2^{\{1,2,3,4\}}$ is defined by the term 
(Def. 13) $\begin{cases}\operatorname{Select} 12-\sigma \text {-field }(k), & \text { if } k \leqslant 2, \\ \Omega_{\text {fut } 2}, & \text { otherwise. }\end{cases}$

Now we state the propositions:

(27) Let us consider a $\sigma$-field $\Sigma$ of subsets of $\{1,2,3,4\}$, and a set $I$. Suppose $I=\{1,2,3\}$ and $\Sigma=2^{\{1,2,3,4\}}$. Then there exists a many sorted $\sigma$-field $M$ over $I$ and $\Sigma$ such that

(i) $M(1)=\Omega_{\text {now }}$, and

(ii) $M(2)=\Omega_{f u t 1}$, and

(iii) $M(3)=\Omega_{\text {fut } 2}$.

Proof: Define $\mathcal{U}$ (element of $\{1,2,3\})=\operatorname{Select} 123-\sigma$-field $\left(\$_{1}\right)$. Consider $f_{4}$ being a function from $\{1,2,3\}$ into $2^{2^{\{1,2,3,4\}}}$ such that for every element $d$ of $\{1,2,3\}, f_{4}(d)=\mathcal{U}(d)$ from [5, Sch. 4]. For every set $i$ such that $i \in I$ holds $f_{4}(i)$ is a $\sigma$-field of subsets of $\{1,2,3,4\}$.

(28) Let us consider a non empty set $\Omega$, a $\sigma$-field $\Sigma$ of subsets of $\Omega$, and a non empty subset $I$ of $\mathbb{R}$. Suppose $I=\{1,2,3\}$ and $\Sigma=2^{\{1,2,3,4\}}$ and $\Omega=\{1,2,3,4\}$. Then there exists a many sorted $\sigma$-field $M$ over $I$ and $\Sigma$ such that

(i) $M(1)=\Omega_{\text {now }}$, and

(ii) $M(2)=\Omega_{f u t 1}$, and

(iii) $M(3)=\Omega_{f u t 2}$, and

(iv) $M$ is a filtration of $I$ and $\Sigma$.

The theorem is a consequence of (27).

(29) Let us consider a non empty set $\Omega$, a $\sigma$-field $\Sigma$ of subsets of $\Omega$, and a $\sigma$-field $\Sigma_{2}$ of subsets of $\{1\}$. Suppose $\Omega=\{1,2,3,4\}$. Then there exists a function $X_{1}$ from $\Omega$ into $\{1\}$ such that $X_{1}$ is random variable of $\Omega_{\text {now }}$ and $\Sigma_{2}$, random variable of $\Omega_{f u t 1}$ and $\Sigma_{2}$, and random variable of $\Omega_{f u t 2}$ and $\Sigma_{2}$.

(30) Let us consider a non empty set $\Omega$, a $\sigma$-field $\Sigma$ of subsets of $\Omega$, and a non empty subset $I$ of $\mathbb{R}$. Suppose $I=\{1,2,3\}$ and $\Sigma=2^{\{1,2,3,4\}}$ and $\Omega=\{1,2,3,4\}$. Then there exists a many sorted $\sigma$-field $M$ over $I$ and $\Sigma$ such that

(i) $M(1)=\Omega_{\text {now }}$, and

(ii) $M(2)=\Omega_{f u t 1}$, and

(iii) $M(3)=\Omega_{f u t 2}$, and

(iv) $M$ is a filtration of $I$ and $\Sigma$.

The theorem is a consequence of (27). 
(31) There exist non empty sets $\Omega, \Omega_{2}$ and there exists a $\sigma$-field $\Sigma$ of subsets of $\Omega$ and there exists a $\sigma$-field $\Sigma_{2}$ of subsets of $\Omega_{2}$ and there exists a non empty subset $I$ of $\mathbb{R}$ and there exists a many sorted $\sigma$-field $Q$ over $I$ and $\Sigma$ such that $Q$ is a filtration of $I$ and $\Sigma$ and there exists a function $\mathcal{R} \mathcal{V}$ from $\Omega$ into $\Omega_{2}$ such that for every element $i$ of $I, \mathcal{R} \mathcal{V}$ is a random variable of $\mathcal{M}_{\sigma \text {-field }}(Q, i)$ and $\Sigma_{2}$. The theorem is a consequence of (30) and (29).

(32) Let us consider non empty sets $\Omega, \Omega_{2}$, a $\sigma$-field $\Sigma$ of subsets of $\Omega$, a $\sigma$ field $\Sigma_{2}$ of subsets of $\Omega_{2}$, a non empty subset $I$ of $\mathbb{R}$, and a filtration $Q$ of $I$ and $\Sigma$. Then there exists a function $\mathcal{R} \mathcal{V}$ from $\Omega$ into $\Omega_{2}$ such that for every element $i$ of $I, \mathcal{R} \mathcal{V}$ is a random variable of $\mathcal{M}_{\sigma \text {-field }}(Q, i)$ and $\Sigma_{2}$.

Proof: Consider $w$ being an object such that $w \in \Omega_{2}$. Set $m_{1}=w$. Consider $m$ being a function from $\Omega$ into $\Omega_{2}$ such that $m=\Omega \longmapsto m_{1}$. For every element $i$ of $I, m$ is a random variable of $\mathcal{M}_{\sigma \text {-field }}(Q, i)$ and $\Sigma_{2}$ by [13, (7)], [11, (5), (4)].

\section{Stochastic Process: Adapted and Predictable}

Now we state the proposition:

(33) Let us consider a non empty set $\Omega$, a $\sigma$-field $\Sigma$ of subsets of $\Omega$, and a $\sigma$ field $\Sigma_{2}$ of subsets of $\Omega$. If $\Sigma_{2} \subseteq \Sigma$, then every event of $\Sigma_{2}$ is an event of $\Sigma$.

Let $\Omega, \Omega_{2}$ be non empty sets, $\Sigma$ be a $\sigma$-field of subsets of $\Omega, \Sigma_{2}$ be a $\sigma$-field of subsets of $\Omega_{2}, I$ be a non empty subset of $\mathbb{R}$, and $P$ be a probability on $\Sigma$.

A stochastic process of $I, \Sigma, \Sigma_{2}$ and $P$ is a function from $I$ into the set of random variables on $\Sigma$ and $\Sigma_{2}$ and is defined by

(Def. 14) for every element $k$ of $I$, there exists a function $\mathcal{R} \mathcal{V}$ from $\Omega$ into $\Omega_{2}$ such that it $(k)=\mathcal{R} \mathcal{V}$ and $\mathcal{R} \mathcal{V}$ is random variable on $\Sigma$ and $\Sigma_{2}$.

Let $S$ be a stochastic process of $I, \Sigma, \Sigma_{2}$ and $P$ and $k$ be an element of $I$. The $k-\mathcal{R} \mathcal{V}$ of $S$ yielding a random variable of $\Sigma$ and $\Sigma_{2}$ is defined by the term (Def. 15) $S(k)$.

An adapted stochastic process of $I, \Sigma, \Sigma_{2}, P$ and $S$ is a function from $I$ into the set of random variables on $\Sigma$ and $\Sigma_{2}$ and is defined by

(Def. 16) there exists a filtration $k$ of $I$ and $\Sigma$ such that for every element $i$ of $I$, the $i-\mathcal{R} \mathcal{V}$ of $S$ is random variable on the $i-\mathcal{E} \mathcal{F}$ of $k$ and $\Sigma_{2}$.

Let $I$ be a non empty subset of $\mathbb{N}, J$ be a non empty subset of $\mathbb{N}$, and $S$ be a stochastic process of $J\left(\in 2^{\mathbb{R}}\right), \Sigma, \Sigma_{2}$ and $P$.

A predictable stochastic process of $I, J, \Sigma, \Sigma_{2}, P$ and $S$ is a function from $J$ into the set of random variables on $\Sigma$ and $\Sigma_{2}$ and is defined by 
(Def. 17) there exists a filtration $k$ of $I\left(\in 2^{\mathbb{R}}\right)$ and $\Sigma$ such that for every element $j$ of $J\left(\in 2^{\mathbb{R}}\right)$ for every element $i$ of $I\left(\in 2^{\mathbb{R}}\right)$ such that $j-1=i$ holds the $j$-R $\mathcal{V}$ of $S$ is random variable on the $i-\mathcal{E} \mathcal{F}$ of $k$ and $\Sigma_{2}$.

Let $I$ be a non empty subset of $\mathbb{R}, M$ be a filtration of $I$ and $\Sigma$, and $S$ be a stochastic process of $I, \Sigma, \Sigma_{2}$ and $P$. We say that $S$ is $M$-stochastic process w.r.t. filtration if and only if

(Def. 18) for every element $i$ of $I$, the $i-\mathcal{R} \mathcal{V}$ of $S$ is random variable on the $i-\mathcal{E F}$ of $M$ and $\Sigma_{2}$.

Now we state the proposition:

(34) Let us consider non empty sets $\Omega, \Omega_{2}$, a $\sigma$-field $\Sigma$ of subsets of $\Omega$, a $\sigma$ field $\Sigma_{2}$ of subsets of $\Omega_{2}$, a non empty subset $I$ of $\mathbb{R}$, a probability $P$ on $\Sigma$, a filtration $M$ of $I$ and $\Sigma$, and a stochastic process $S$ of $I, \Sigma, \Sigma_{2}$ and $P$. Suppose $S$ is $M$-stochastic process w.r.t. filtration. Then $S$ is an adapted stochastic process of $I, \Sigma, \Sigma_{2}, P$ and $S$.

\section{Example for a Stochastic Process}

Let $k_{1}, k_{2}$ be elements of $\mathbb{R}, \Omega$ be a non empty set, and $k$ be an element of $\Omega$. The functors: $\operatorname{Set} 1-\mathcal{R} \mathcal{V}\left(k_{1}, k_{2}, k\right)$ and $\operatorname{Set} 4-\mathcal{R} \mathcal{V}\left(k_{1}, k_{2}, k\right)$ yielding elements of $\mathbb{R}$ are defined by terms

(Def. 19) $\begin{cases}k_{1}, & \text { if } k=1 \text { or } k=2, \\ k_{2}, & \text { otherwise, }\end{cases}$

(Def. 20) $\begin{cases}k_{1}, & \text { if } k=3, \\ k_{2}, & \text { otherwise, }\end{cases}$ respectively. Let $k_{2}, k_{3}, k_{4}$ be elements of $\mathbb{R}$. The functor $\operatorname{Set} 3-\mathcal{R} \mathcal{V}\left(k_{2}, k_{3}, k_{4}, k\right)$ yielding an element of $\mathbb{R}$ is defined by the term

(Def. 21) $\begin{cases}k_{2}, & \text { if } k=2, \\ \operatorname{Set} 4-\mathcal{R} \mathcal{V}\left(k_{3}, k_{4}, k\right), & \text { otherwise. }\end{cases}$

Let $k_{1}, k_{2}, k_{3}, k_{4}$ be elements of $\mathbb{R}$. The functor $\operatorname{Set} 2-\mathcal{R} \mathcal{V}\left(k_{1}, k_{2}, k_{3}, k_{4}, k\right)$ yielding an element of $\mathbb{R}$ is defined by the term

(Def. 22) $\begin{cases}k_{1}, & \text { if } k=1, \\ \operatorname{Set} 3-\mathcal{R} \mathcal{V}\left(k_{2}, k_{3}, k_{4}, k\right), & \text { otherwise. }\end{cases}$

Now we state the proposition:

(35) Let us consider elements $k_{1}, k_{2}, k_{3}, k_{4}$ of $\mathbb{R}$, and a set $\Omega$. Suppose $\Omega=$ $\{1,2,3,4\}$. Then there exists a function $f$ from $\Omega$ into $\mathbb{R}$ such that

(i) $f(1)=k_{1}$, and

(ii) $f(2)=k_{2}$, and 
(iii) $f(3)=k_{3}$, and

(iv) $f(4)=k_{4}$.

Proof: Define $\mathcal{U}$ (element of $\Omega$ ) $=\operatorname{Set} 2-\mathcal{R} \mathcal{V}\left(k_{1}, k_{2}, k_{3}, k_{4}, \$_{1}\right)$. Consider $f$ being a function from $\Omega$ into $\mathbb{R}$ such that for every element $d$ of $\Omega$, $f(d)=\mathcal{U}(d)$ from [5, Sch. 4]. $f(1)=k_{1} \cdot f(2)=k_{2} . f(3)=k_{3} . f(4)=k_{4}$.

Let us consider a set $\Omega$.

Let us assume that $\Omega=\{1,2,3,4\}$. Now we state the propositions:

(36) There exists a function $f$ from $\Omega$ into $\mathbb{R}$ such that

(i) $f(1)=100$, and

(ii) $f(2)=100$, and

(iii) $f(3)=100$, and

(iv) $f(4)=100$.

The theorem is a consequence of (35).

(37) There exists a function $f$ from $\Omega$ into $\mathbb{R}$ such that

(i) $f(1)=80$, and

(ii) $f(2)=80$, and

(iii) $f(3)=120$, and

(iv) $f(4)=120$.

The theorem is a consequence of (35).

(38) There exists a function $f$ from $\Omega$ into $\mathbb{R}$ such that

(i) $f(1)=60$, and

(ii) $f(2)=80$, and

(iii) $f(3)=100$, and

(iv) $f(4)=120$.

The theorem is a consequence of (35).

(39) Let us consider elements $k_{1}, k_{2}, k_{3}, k_{4}$ of $\mathbb{R}$, and a non empty set $\Omega$. Suppose $\Omega=\{1,2,3,4\}$. Let us consider a $\sigma$-field $\Sigma$ of subsets of $\Omega$, a non empty subset $I$ of $\mathbb{R}$, and a filtration $M$ of $I$ and $\Sigma$. Suppose $M(1)=\Omega_{\text {now }}$ and $M(2)=\Omega_{f u t 1}$ and $M(3)=\Omega_{f u t 2}$. Let us consider an element $k$ of $I$. Suppose $k=3$. Then there exists a function $f$ from $\Omega$ into $\mathbb{R}$ such that

(i) $f(1)=k_{1}$, and

(ii) $f(2)=k_{2}$, and

(iii) $f(3)=k_{3}$, and 
(iv) $f(4)=k_{4}$, and

(v) $f$ is random variable on the $k-\mathcal{E} \mathcal{F}$ of $M$ and the Borel sets.

Proof: Consider $f$ being a function from $\Omega$ into $\mathbb{R}$ such that $f(1)=k_{1}$ and $f(2)=k_{2}$ and $f(3)=k_{3}$ and $f(4)=k_{4} .1,2,3,4 \in \operatorname{dom} f . f$ is random variable on the $k-\mathcal{E F}$ of $M$ and the Borel sets by [4, (1)], [11, (4)].

Let us consider a non empty set $\Omega$, a $\sigma$-field $\Sigma$ of subsets of $\Omega$, a non empty subset $I$ of $\mathbb{R}$, a filtration $M$ of $I$ and $\Sigma$, and an element $k$ of $I$.

Let us assume that $\Omega=\{1,2,3,4\}$. Now we state the propositions:

(40) Suppose $M(1)=\Omega_{\text {now }}$ and $M(2)=\Omega_{\text {fut } 1}$ and $M(3)=\Omega_{\text {fut } 2}$. Then suppose $k=3$. Then there exists a function $f$ from $\Omega$ into $\mathbb{R}$ such that

(i) $f(1)=60$, and

(ii) $f(2)=80$, and

(iii) $f(3)=100$, and

(iv) $f(4)=120$, and

(v) $f$ is random variable on the $k-\mathcal{E} \mathcal{F}$ of $M$ and the Borel sets.

The theorem is a consequence of (39).

(41) Suppose $M(1)=\Omega_{\text {now }}$ and $M(2)=\Omega_{f u t 1}$ and $M(3)=\Omega_{f u t 2}$. Then suppose $k=3$. Then there exists a function $f$ from $\Omega$ into $\mathbb{R}$ such that

(i) $f(1)=180$, and

(ii) $f(2)=120$, and

(iii) $f(3)=120$, and

(iv) $f(4)=80$, and

(v) $f$ is random variable on the $k-\mathcal{E F}$ of $M$ and the Borel sets.

The theorem is a consequence of (39).

(42) Let us consider elements $k_{1}, k_{2}$ of $\mathbb{R}$, and a non empty set $\Omega$. Suppose $\Omega=\{1,2,3,4\}$. Let us consider a $\sigma$-field $\Sigma$ of subsets of $\Omega$, a non empty subset $I$ of $\mathbb{R}$, and a filtration $M$ of $I$ and $\Sigma$. Suppose $M(1)=\Omega_{\text {now }}$ and $M(2)=\Omega_{f u t 1}$ and $M(3)=\Omega_{f u t 2}$. Let us consider an element $k$ of $I$. Suppose $k=2$. Then there exists a function $f$ from $\Omega$ into $\mathbb{R}$ such that

(i) $f(1)=k_{1}$, and

(ii) $f(2)=k_{1}$, and

(iii) $f(3)=k_{2}$, and

(iv) $f(4)=k_{2}$, and

(v) $f$ is random variable on the $k-\mathcal{E} \mathcal{F}$ of $M$ and the Borel sets. 
Proof: Consider $f$ being a function from $\Omega$ into $\mathbb{R}$ such that $f(1)=k_{1}$ and $f(2)=k_{1}$ and $f(3)=k_{2}$ and $f(4)=k_{2}$. Set $i=k$. For every set $x$, $f^{-1}(x) \in$ the $i-\mathcal{E} \mathcal{F}$ of $M$ by [4, (1)].

Let us consider a non empty set $\Omega$, a $\sigma$-field $\Sigma$ of subsets of $\Omega$, a non empty subset $I$ of $\mathbb{R}$, a filtration $M$ of $I$ and $\Sigma$, and an element $k$ of $I$.

Let us assume that $\Omega=\{1,2,3,4\}$. Now we state the propositions:

(43) Suppose $M(1)=\Omega_{\text {now }}$ and $M(2)=\Omega_{f u t 1}$ and $M(3)=\Omega_{f u t 2}$. Then suppose $k=2$. Then there exists a function $f$ from $\Omega$ into $\mathbb{R}$ such that

(i) $f(1)=80$, and

(ii) $f(2)=80$, and

(iii) $f(3)=120$, and

(iv) $f(4)=120$, and

(v) $f$ is random variable on the $k-\mathcal{E} \mathcal{F}$ of $M$ and the Borel sets.

The theorem is a consequence of (42).

(44) Suppose $M(1)=\Omega_{\text {now }}$ and $M(2)=\Omega_{f u t 1}$ and $M(3)=\Omega_{f u t 2}$. Then suppose $k=2$. Then there exists a function $f$ from $\Omega$ into $\mathbb{R}$ such that

(i) $f(1)=150$, and

(ii) $f(2)=150$, and

(iii) $f(3)=100$, and

(iv) $f(4)=100$, and

(v) $f$ is random variable on the $k-\mathcal{E} \mathcal{F}$ of $M$ and the Borel sets.

The theorem is a consequence of (42).

(45) Let us consider an element $k_{1}$ of $\mathbb{R}$, and a non empty set $\Omega$. Suppose $\Omega=\{1,2,3,4\}$. Let us consider a $\sigma$-field $\Sigma$ of subsets of $\Omega$, a non empty subset $I$ of $\mathbb{R}$, and a filtration $M$ of $I$ and $\Sigma$. Suppose $M(1)=\Omega_{\text {now }}$ and $M(2)=\Omega_{f u t 1}$ and $M(3)=\Omega_{f u t 2}$. Let us consider an element $k$ of $I$. Suppose $k=1$. Then there exists a function $f$ from $\Omega$ into $\mathbb{R}$ such that

(i) $f(1)=k_{1}$, and

(ii) $f(2)=k_{1}$, and

(iii) $f(3)=k_{1}$, and

(iv) $f(4)=k_{1}$, and

(v) $f$ is random variable on the $k-\mathcal{E F}$ of $M$ and the Borel sets.

Proof: Consider $f$ being a function from $\Omega$ into $\mathbb{R}$ such that $f(1)=k_{1}$ and $f(2)=k_{1}$ and $f(3)=k_{1}$ and $f(4)=k_{1}$. Set $i=k$. For every set $x$ such that $x \in$ the Borel sets holds $f^{-1}(x) \in$ the $i-\mathcal{E} \mathcal{F}$ of $M$ by [4, (1)]. 
Let us consider a non empty set $\Omega$, a $\sigma$-field $\Sigma$ of subsets of $\Omega$, a non empty subset $I$ of $\mathbb{R}$, a filtration $M$ of $I$ and $\Sigma$, and an element $k$ of $I$.

Let us assume that $\Omega=\{1,2,3,4\}$. Now we state the propositions:

(46) Suppose $M(1)=\Omega_{\text {now }}$ and $M(2)=\Omega_{f u t 1}$ and $M(3)=\Omega_{f u t 2}$. Then suppose $k=1$. Then there exists a function $f$ from $\Omega$ into $\mathbb{R}$ such that

(i) $f(1)=100$, and

(ii) $f(2)=100$, and

(iii) $f(3)=100$, and

(iv) $f(4)=100$, and

(v) $f$ is random variable on the $k-\mathcal{E F}$ of $M$ and the Borel sets.

The theorem is a consequence of (45).

(47) Suppose $M(1)=\Omega_{\text {now }}$ and $M(2)=\Omega_{f u t 1}$ and $M(3)=\Omega_{\text {fut } 2 \text {. Then }}$ suppose $k=1$. Then there exists a function $f$ from $\Omega$ into $\mathbb{R}$ such that

(i) $f(1)=125$, and

(ii) $f(2)=125$, and

(iii) $f(3)=125$, and

(iv) $f(4)=125$, and

(v) $f$ is random variable on the $k-\mathcal{E F}$ of $M$ and the Borel sets.

The theorem is a consequence of (45).

Now we state the proposition:

(48) Let us consider a non empty set $\Omega$. Suppose $\Omega=\{1,2,3,4\}$. Let us consider a $\sigma$-field $\Sigma$ of subsets of $\Omega$, and a non empty subset $I$ of $\mathbb{R}$. Suppose $I=\{1,2,3\}$ and $\Sigma=2^{\{1,2,3,4\}}$. Let us consider a filtration $M$ of $I$ and $\Sigma$. Suppose $M(1)=\Omega_{\text {now }}$ and $M(2)=\Omega_{f u t 1}$ and $M(3)=\Omega_{f u t 2}$. Let us consider a probability $P$ on $\Sigma$, and an element $i$ of $I$. Then there exists a function $\mathcal{R} \mathcal{V}$ from $\Omega$ into $\mathbb{R}$ such that $\mathcal{R} \mathcal{V}$ is random variable on the $i-\mathcal{E F}$ of $M$ and the Borel sets. The theorem is a consequence of (46), (43), and (40).

Let $I$ be a non empty subset of $\mathbb{R}$. Assume $I=\{1,2,3\}$. Let $i$ be an element of $I$. Assume $i=2$ or $i=3$. Let $\Omega$ be a non empty set. Assume $\Omega=\{1,2,3,4\}$. Let $\Sigma$ be a $\sigma$-field of subsets of $\Omega$. Assume $\Sigma=2^{\Omega}$. Let $f_{1}$ be a function from $\Omega$ into $\mathbb{R}$. Assume $f_{1}(1)=60$ and $f_{1}(2)=80$ and $f_{1}(3)=100$ and $f_{1}(4)=120$. Let $f_{2}$ be a function from $\Omega$ into $\mathbb{R}$. Assume $f_{2}(1)=80$ and $f_{2}(2)=80$ and $f_{2}(3)=120$ and $f_{2}(4)=120$. Let $f_{3}$ be a function from $\Omega$ into $\mathbb{R}$. The functor Select12- $\mathcal{R} \mathcal{V}\left(i, \Sigma, f_{1}, f_{2}, f_{3}\right)$ yielding an element of the set of random variables on $\Sigma$ and the Borel sets is defined by the term 
(Def. 23) $\begin{cases}f_{2}, & \text { if } i=2, \\ f_{1}, & \text { otherwise. }\end{cases}$

Assume $I=\{1,2,3\}$. Assume $\Omega=\{1,2,3,4\}$. Assume $\Sigma=2^{\Omega}$. Let $f_{1}, f_{2}$ be functions from $\Omega$ into $\mathbb{R}$. Assume $f_{3}(1)=100$ and $f_{3}(2)=100$ and $f_{3}(3)=100$ and $f_{3}(4)=100$. The functor Select123- $\mathcal{R} \mathcal{V}\left(i, \Sigma, f_{1}, f_{2}, f_{3}\right)$ yielding an element of the set of random variables on $\Sigma$ and the Borel sets is defined by the term

(Def. 24) $\begin{cases}\operatorname{Select} 12-\mathcal{R} \mathcal{V}\left(i, \Sigma, f_{1}, f_{2}, f_{3}\right), & \text { if } i=2 \text { or } i=3, \\ f_{3}, & \text { otherwise. }\end{cases}$

Now we state the proposition:

(49) Let us consider non empty sets $\Omega, \Omega_{2}$. Suppose $\Omega=\{1,2,3,4\}$. Let us consider a $\sigma$-field $\Sigma$ of subsets of $\Omega$, and a non empty subset $I$ of $\mathbb{R}$. Suppose $I=\{1,2,3\}$ and $\Sigma=2^{\{1,2,3,4\}}$. Let us consider a probability $P$ on $\Sigma$, and a filtration $M$ of $I$ and $\Sigma$. Suppose $M(1)=\Omega_{\text {now }}$ and $M(2)=\Omega_{f u t 1}$ and $M(3)=\Omega_{f u t 2}$. Then there exists a stochastic process $S$ of $I, \Sigma$, the Borel sets and $P$ such that

(i) for every element $k$ of $I$, there exists a function $\mathcal{R} \mathcal{V}$ from $\Omega$ into $\mathbb{R}$ such that $S(k)=\mathcal{R} \mathcal{V}$ and $\mathcal{R} \mathcal{V}$ is random variable on $\Sigma$ and the Borel sets and random variable on the $k-\mathcal{E F}$ of $M$ and the Borel sets and there exists a function $f$ from $\Omega$ into $\mathbb{R}$ such that if $k=1$, then $f(1)=100$ and $f(2)=100$ and $f(3)=100$ and $f(4)=100$ and $S(k)=f$ and there exists a function $f$ from $\Omega$ into $\mathbb{R}$ such that if $k=2$, then $f(1)=80$ and $f(2)=80$ and $f(3)=120$ and $f(4)=120$ and $S(k)=f$ and there exists a function $f$ from $\Omega$ into $\mathbb{R}$ such that if $k=3$, then $f(1)=60$ and $f(2)=80$ and $f(3)=100$ and $f(4)=120$ and $S(k)=f$ and $S$ is $M$-stochastic process w.r.t. filtration, and

(ii) $S$ is an adapted stochastic process of $I, \Sigma$, the Borel sets, $P$ and $S$. Proof: Consider $f_{3}$ being a function from $\Omega$ into $\mathbb{R}$ such that $f_{3}(1)=$ 100 and $f_{3}(2)=100$ and $f_{3}(3)=100$ and $f_{3}(4)=100$. Consider $f_{2}$ being a function from $\Omega$ into $\mathbb{R}$ such that $f_{2}(1)=80$ and $f_{2}(2)=80$ and $f_{2}(3)=120$ and $f_{2}(4)=120$. Consider $f_{1}$ being a function from $\Omega$ into $\mathbb{R}$ such that $f_{1}(1)=60$ and $f_{1}(2)=80$ and $f_{1}(3)=100$ and $f_{1}(4)=120$. Define $\mathcal{U}($ element of $I)=$ Select $123-\mathcal{R} \mathcal{V}\left(\$_{1}, \Sigma, f_{1}, f_{2}, f_{3}\right)$. Consider $f_{4}$ being a function from $I$ into the set of random variables on $\Sigma$ and the Borel sets such that for every element $d$ of $I, f_{4}(d)=\mathcal{U}(d)$ from [5, Sch. 4]. For every element $k$ of $I$, there exists a function $\mathcal{R} \mathcal{V}$ from $\Omega$ into $\mathbb{R}$ such that $f_{4}(k)=\mathcal{R} \mathcal{V}$ and $\mathcal{R} \mathcal{V}$ is random variable on $\Sigma$ and the Borel sets. For every element $k$ of $I$, there exists a function $\mathcal{R} \mathcal{V}$ from $\Omega$ into $\mathbb{R}$ such that $f_{4}(k)=\mathcal{R} \mathcal{V}$ and $\mathcal{R} \mathcal{V}$ is random variable on $\Sigma$ and the Borel sets and random variable on the $k-\mathcal{E F}$ of $M$ and the Borel sets and there exists 
a function $f$ from $\Omega$ into $\mathbb{R}$ such that if $k=1$, then $f(1)=100$ and $f(2)=100$ and $f(3)=100$ and $f(4)=100$ and $f_{4}(k)=f$ and there exists a function $f$ from $\Omega$ into $\mathbb{R}$ such that if $k=2$, then $f(1)=80$ and $f(2)=80$ and $f(3)=120$ and $f(4)=120$ and $f_{4}(k)=f$ and there exists a function $f$ from $\Omega$ into $\mathbb{R}$ such that if $k=3$, then $f(1)=60$ and $f(2)=80$ and $f(3)=100$ and $f(4)=120$ and $f_{4}(k)=f$ and $f_{4}$ is $M$-stochastic process w.r.t. filtration and adapted stochastic process of $I$, $\Sigma$, the Borel sets, $P$ and $f_{4}$.

\section{REFERENCES}

[1] Grzegorz Bancerek. The fundamental properties of natural numbers Formalized Mathematics, 1(1):41-46, 1990.

[2] Grzegorz Bancerek, Czesław Byliński, Adam Grabowski, Artur Korniłowicz, Roman Matuszewski, Adam Naumowicz, Karol Pąk, and Josef Urban. Mizar: State-of-the-art and beyond. In Manfred Kerber, Jacques Carette, Cezary Kaliszyk, Florian Rabe, and Volker Sorge, editors, Intelligent Computer Mathematics, volume 9150 of Lecture Notes in Computer Science, pages 261-279. Springer International Publishing, 2015. ISBN 978-3319-20614-1. doi 10.1007/978-3-319-20615-8_17.

[3] Francesca Biagini and Daniel Rost. Money out of nothing? - Prinzipien und Grundlagen der Finanzmathematik. MATHE-LMU.DE, LIMU-Munchen(25):28-34, 2012.

[4] Czesław Byliński. Functions and their basic properties Formalized Mathematics, 1(1): 55-65, 1990.

[5] Czesław Byliński. Functions from a set to a set Formalized Mathematics, 1(1):153-164, 1990.

[6] Hans Föllmer and Alexander Schied. Stochastic Finance: An Introduction in Discrete Time, volume 27 of Studies in Mathematics. de Gruyter, Berlin, 2nd edition, 2004.

[7] Hans-Otto Georgii. Stochastik, Einführung in die Wahrscheinlichkeitstheorie und Statistik. deGruyter, Berlin, 2nd edition, 2004.

[8] Peter Jaeger. Events of Borel sets, construction of Borel sets and random variables for stochastic finance. Formalized Mathematics, 22(3):199-204, 2014. doi 10.2478/forma2014-0022.

[9] Achim Klenke. Wahrscheinlichkeitstheorie. Springer-Verlag, Berlin, Heidelberg, 2006.

[10] Jürgen Kremer. Einführung in die diskrete Finanzmathematik. Springer-Verlag, Berlin, Heidelberg, New York, 2006.

[11] Andrzej Nędzusiak. $\sigma$-fields and probability Formalized Mathematics, 1(2):401-407, 1990.

[12] Klaus Sandmann. Einführung in die Stochastik der Finanzmärkte. Springer-Verlag, Berlin, Heidelberg, New York, 2 edition, 2001.

[13] Andrzej Trybulec. Binary operations applied to functions Formalized Mathematics, 1 (2):329-334, 1990.

Received December 30, 2015 